\title{
Clustering Characteristics of Millimeter Wave Indoor Channels
}

\author{
Behnam Neekzad $\dagger$, Kamran Sayrafian-Pour*, John S. Baras $\dagger$ \\ $\dagger$ Electrical \& Computer Engineering Department *Information Technology Laboratory \\ University of Maryland \\ College Park, MD, 20742 \\ National Institute of Standard \& Technology \\ Gaithersburg, MD, 20899
}

\begin{abstract}
Temporal-Angular channel sounding measurements of an indoor millimeter wave channel $(60 \mathrm{GHz})$ is analyzed to determine whether ray arrivals at the receiver form clusters in the two-dimensional time-angle space. The channel behavior and cluster energy distribution for directional antennas (with various 3dB beamwidths) at the transmitter are discussed. Geometric interpretation of the results is also provided.
\end{abstract}

Index Terms-Channel characterization, Millimeter wave propagation. Temporal-Angular clustering

\section{INTRODUCTION}

Millimeter wave technology is becoming increasingly important in many military and commercial applications. Remote sensing, radio astronomy, passive imaging, radar and high data rate communication are among these applications. To establish a high data rate communication link between two nodes, acceptable Signal to Noise Ratio (SNR) at the receiver is required. However, due to the high propagation path loss and signal attenuation that is associated with the millimeter wave signal, it is extremely valuable to have knowledge of how millimeter wave signals propagate in the environment. Such information along with appropriate smart antenna technology could enable a system to maximize the quality of the received signal and therefore, achieve higher throughput.

Indoor environments create challenging multipath propagation scenarios with significant time and angular spread. Joint temporal-angular clustering phenomenon have been observed at frequencies commonly used in wireless networks and 2-D statistical channel models have been proposed based on such phenomenon [1], [2]. However, at millimeter wave frequencies, and to the best of our knowledge, no reports have been made for such clustering behavior. The clustering of arrival rays can have a significant impact on channel capacity. Un-clustered models tend to overestimate the capacity if the multipath components are indeed clustered [14].

Considering angular (i.e. spatial) aspects of indoor channel is extremely important for systems employing multiple antennas. Millimeter wave wireless communication systems can greatly benefit from this knowledge as the physical size of array antennas makes them amenable for practical implementation [15]. Knowledge of cluster locations is especially useful for such systems as it could be exploited by various spatial diversity combining or beamforming algorithms to enhance the system's performance [18].
The objective of this paper is to study the 2-D clustering phenomenon of millimeter wave propagation $(60 \mathrm{GHz})$ at indoor environments and particularly observe the effect of directional antennas with various beamwidths at the transmitter on the formation and energy distribution of such clusters.

The rest of this paper is organized as follows. Measurement setup and the corresponding environments will be described in section 2. In section 3, we will outline data analysis and processing methodology. Results are provided in section 4 and finally, conclusions will be discussed in Section 5.

\section{MEASUREMENT SETUP AND ENVIRONMENTS}

Field measurements have been conducted in [3] for both residential and office environments. The residential environment is a Line of Sight (LOS) scenario as shown in Fig. 1. The room where the measurements took place has no furniture and a LOS path exists between the transmitter and receiver. The floor, ceiling and the door are made of wood. The surface of the walls and ceiling is covered with wallpaper. There are also 3 large windows (plane glass) on the two intersecting walls. The height of the ceiling and the window is $2.47 \mathrm{~m}$ and $2.11 \mathrm{~m}$ respectively. The transmitter and receiver are located $1.1 \mathrm{~m}$ above the floor.

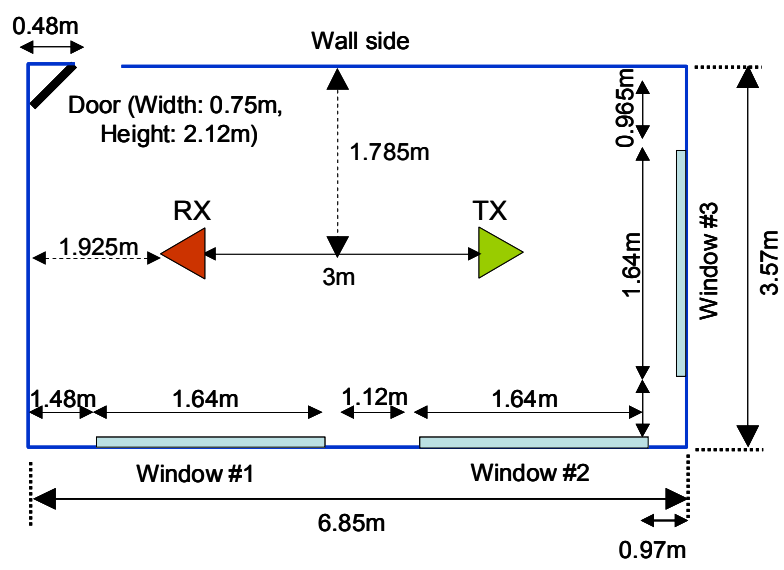

Figure 1: Layout of the residential room

The office environment is a Non-Line of Sight (NLOS) scenario as shown in Fig. 2. The room is made of steel wall, steel ceiling and steel floor. The floor and the ceiling are covered with carpet and plaster board, respectively. Glass windows exist on one side of the office. To obtain the angular component of the received millimeter wave signal, a 
directional antenna with a narrow angular resolution of 15 degrees was used at the receiver. The antenna was positioned to face the transmitter initially and then mechanically rotated in steps of $5^{\circ}$ until full $360^{\circ}$ was obtained.

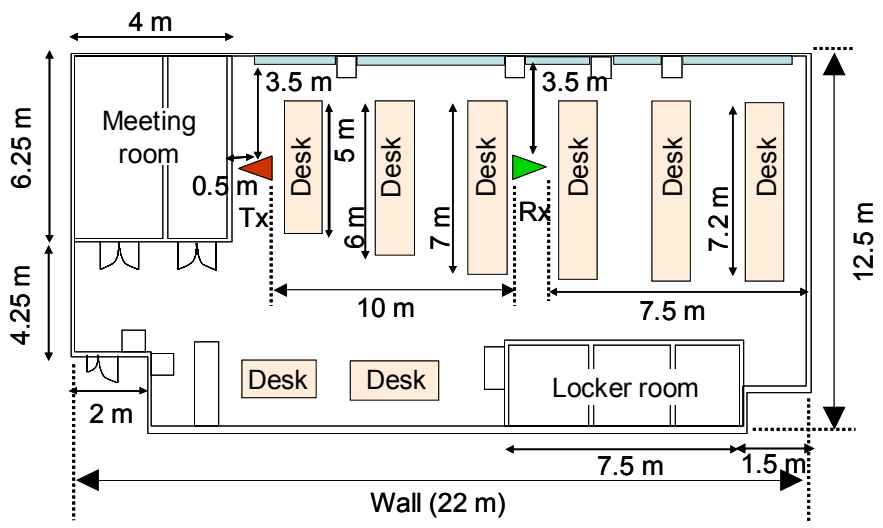

Figure 2: Layout of the office

A Vector Network Analyzer (VNA) was used as the signal generator and receiver. All measurements were conducted in the frequency domain using the VNA. The receiver antenna used in [3] is a pyramid horn antenna with $22 \mathrm{dBi}$ gain (see Fig. 3 ). The $3 \mathrm{~dB}$ beamwidth of the antenna in horizontal direction is $15^{\circ}$. At the transmitter, in addition to an omni-directional antenna, various pyramid horn antennas with 10,16 and 22 $\mathrm{dBi}$ gains were used for the measurements. These are equivalent to $60^{\circ}, 30^{\circ}$, and $15^{\circ} 3-\mathrm{dB}$ beamwidths. Calibration was performed in an anechoic chamber with one meter reference distance to remove the antenna effects.

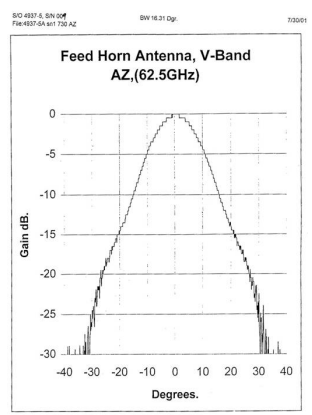

(a)

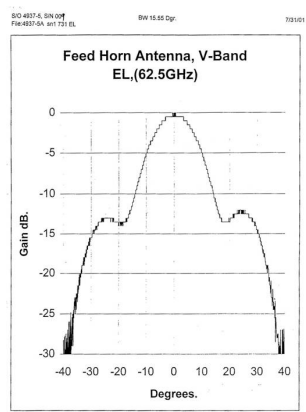

(b)
Figure 3: Pyramidal horn antenna pattern at the receiver (a) H-plane (b) E-plane

During the measurements, the VNA was set to sequentially transmit 401 (801 for the office) continuous waves tones uniformly distributed over the frequency range $61-64 \mathrm{GHz}$ which results in frequency steps of $7.5 \mathrm{MHz}(3.75 \mathrm{MHz}$ for the office). This frequency resolution gives maximum excess delay of about $133.3 \mathrm{~ns}$ (266.7 ns for the office). The $3 \mathrm{GHz}$ bandwidth results in a temporal resolution of $0.25 \mathrm{~ns}$.

An example of the raw data produced by this setup is shown in Fig. 4. This is a 3-D plot of the received power with respect to time and azimuth angle. The frequency band for the experiment was 61-64 GHz. In order to reduce the effect of non-stationarity in the channel and improve the signal-to-noise ratio, 128 measurements were taken for each angle and the results were averaged.

\section{DATA ANALYSIS AND PROCESSING}

\section{A. CLEAN}

As mentioned in the previous section, the process of measuring angular data involves rotating the directional antenna at the receiver in steps of $5^{\circ}$ after each measurement. This is equivalent to circularly sliding the antenna beam pattern around the $360^{\circ}$ field of view in the azimuth dimension. Noting that the $3 \mathrm{~dB}$ beamwidth of the receiver's directional antenna is $15^{\circ}$, the actual channel data will be affected by this process in a way that is similar to circular convolution.

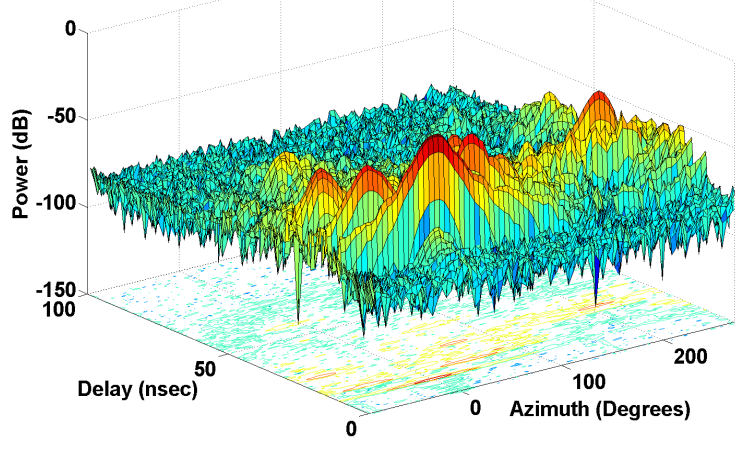

Figure 4: Example of raw data produced by the measurement

Also, the windowing and pulse shaping functions at the receiver impacts the temporal response of the actual channel data. Considering the effects of the measuring process and instruments, the resulting 2-D angular-temporal data can be modeled as the true channel response blurred by a Point Spread Function (PSF). PSF is the measurement system's 2-D angular-temporal impulse response when there is no multipath. Here, the underlying assumption is that specular components are the main significant components of the multipath channel. This is a valid assumption since, as will be seen later, scattered multipath components are negligible and most of the propagated energy is highly localized in time-angle space. The raw measurement data, as a result, represents arrivals rays that have been blurred by the PSF and corrupted by noise at the receiver. The PSF for the measurement experiment discussed in the previous section is displayed in Figure 5.

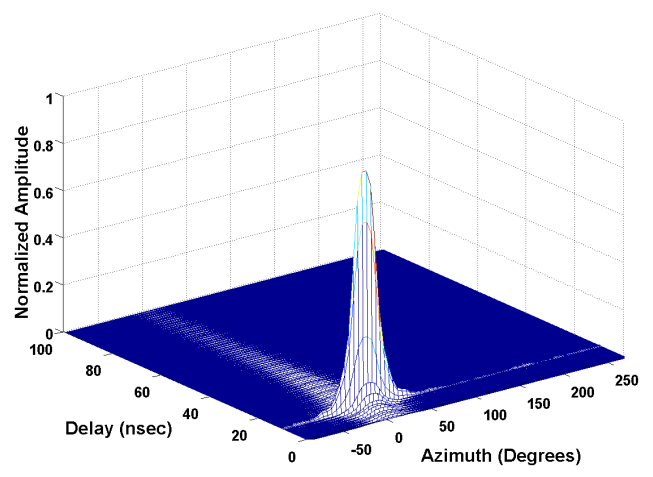

Figure 5: Normalized 2-D PSF

The side-lobes along the time and angle dimensions are due to the effects of windowing, pulse shaping and beam-pattern of the antenna respectively. PSF basically represents how a single 
arrival at the receiver is displayed by the measurement system. Removing the effect of the PSF and therefore identifying the exact times and angles of arrival is consequently equivalent to a two-dimensional (2-D) de-convolution. To solve this problem, we tried different techniques such as LucyRichardson, CLEAN and blind deconvolution. Blind deconvolution [4] did not perform well and it is usually used in situations when complete knowledge of the PSF is not available. The Lucy-Richardson [5], [6] algorithm assumes that the observed data is the result of a perfect convolution with a PSF added to a random noise with Poisson distribution. The CLEAN algorithm is commonly used for processing astronomical images [7] where it is assumed that the radio sky can be represented by a number of point sources in an otherwise empty field of view. Therefore, the resulting image is modeled as groups of point sources convolved with a blurring function that is due to the limitation of the imaging apparatus.

In comparison to the other two methodologies, CLEAN displayed a better performance; therefore, we used this technique to remove the angular-temporal effect of the measuring instrument from the raw data. Implementation-wise, the CLEAN algorithm essentially turns into a recursive subtraction of the shifted PSF from the raw data $[8,9]$. We processed the data for each scenario to remove the effect of the antenna pattern and other processing at the receiver. For cases where there is a directional antenna at the transmitter, it is important to note that the 'CLEAN-ed' data still has the effect of the transmitter's antenna pattern. Therefore, the observed channel characteristics will be dependent on that particular beam pattern.

\section{B Cluster Identification Methodology}

Kernel Density Estimation (KDE) is a nonparametric Probability Density Function (PDF) estimation approach that can be applied to a given set of measured data [10], [11]. KDE is a robust, simple and convenient method to estimate the PDF of a random variable given its sample realization. We have used the two dimensional version of this technique in order to estimate the temporal-angular distribution function of the ray arrivals at the receiver. Having this distribution function and using an appropriate threshold, all 2-D clusters (i.e. temporalangular) can be easily identified.

Let $(t, \theta)$ denote the random variable that represents the time and angle of arrival of a ray at the receiver. If $f(t, \theta)$ is the 2-D PDF of these arrivals, then the multivariate kernel density estimate of this function (i.e. $\hat{f}(t, \theta))$ can be expressed by:

$$
\hat{f}(t, \theta)=\frac{1}{N h_{t} h_{\theta}} \sum_{i=1}^{N} K\left(\frac{(t, \theta)-\left(t_{i}, \theta_{i}\right)}{h_{t} h_{\theta}}\right)
$$

Where $h_{t}$ and $h_{\theta}$ are the temporal and angular bandwidths respectively; $K(x, y)$ is the kernel function, $\left(t_{i}, \theta_{i}\right) i=1,2, \ldots ., N$ are sample realizations of the random variable $(t, \theta)$ (i.e. arrivals) with the unknown density function $f(t, \theta)$ and $N$ is the number of samples. Here, we have two sets of samples which have been obtained through measurement and simulation.

The kernel function is often selected to be a PDF that is symmetric both in time and angle [12]. In our study, we have used the 2-D Normal distribution to be the kernel function as written below:

$$
K(x, y)=\frac{1}{2 \pi} \exp \left(-\frac{1}{2}\|(x, y)\|^{2}\right)
$$

Therefore, the kernel density estimate of $f(t, a)$ will be:

$$
\hat{f}(t, \theta)=\frac{1}{N h_{t} h_{\theta}} \sum_{i=1}^{N} \frac{1}{2 \pi} \exp \left(\frac{-\left\|(t, \theta)-\left(t_{i}, \theta_{i}\right)\right\|^{2}}{2 h_{t} h_{\theta}}\right)
$$

Using this approach, we have processed the 'CLEAN-ed' time-angle impulse response of the channel obtained through measurement. In the next section, we provide the results of this analysis and discuss their geometric relevance.

\section{RESULTS}

One dimensional temporal clustering phenomenon has been observed in indoor channels and wideband statistical channel models have been proposed accordingly. Here, we also observe that arrivals come in few groups (i.e. clusters) scattered in different coordinates throughout the time-angle space. These 2-D clusters are identified by using the KDE and an appropriate threshold representing the noise floor of the receiver.

\section{A. LOS Residential Environment}

Figure 6a displays the clusters obtained by processing the measurement data when an omni-directional antenna is used in the residential environment. A total of 6 clusters are observed in the channel data. The coordinate of these clusters are listed in Table 1. A cluster time (angle) coordinate is defined to be the median of time (angle) of all arrival rays in that cluster.

Table 1: Cluster coordinates for the residential environments

\begin{tabular}{|c|c|c|}
\hline Cluster \# & $\begin{array}{c}\text { Cluster Arrival Angel } \\
\text { (Degrees) }\end{array}$ & $\begin{array}{c}\text { Cluster Arrival Time } \\
\text { (nsec) }\end{array}$ \\
\hline 1 & $0^{\circ}$ & 10 \\
\hline 2 & $-50^{\circ}$ & 15.5 \\
\hline 3 & $50^{\circ}$ & 15.5 \\
\hline 4 & $-74^{\circ}$ & 25.75 \\
\hline 5 & $74^{\circ}$ & 25.75 \\
\hline 6 & $207.5^{\circ}$ & 25.75 \\
\hline
\end{tabular}

A comprehensive geometric interpretation of these results can be provided by considering the main paths that the RF signal travels between the transmitter and receiver [19]. Each cluster formation is the result of a unique path between the transmitter and receiver. These paths which include direct LOS and various single-reflected and double-reflected signals have been graphically identified in Figure 7. There are no clusters due to reflections from the walls behind the transmitter or receiver. This is because that the transmitter and receiver are positioned at the same height and the measuring equipment 


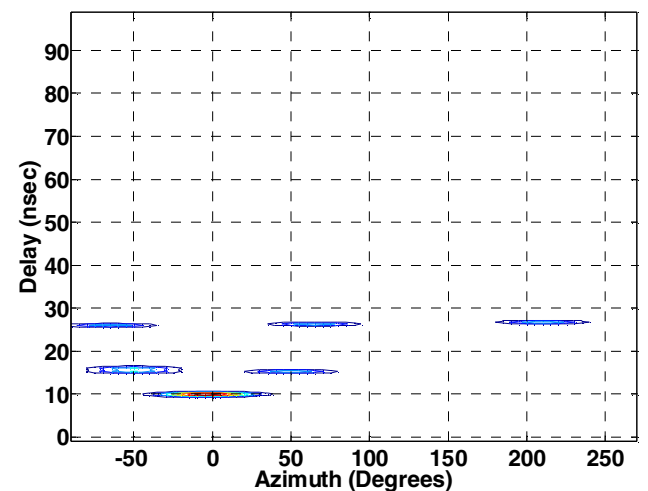

(a)

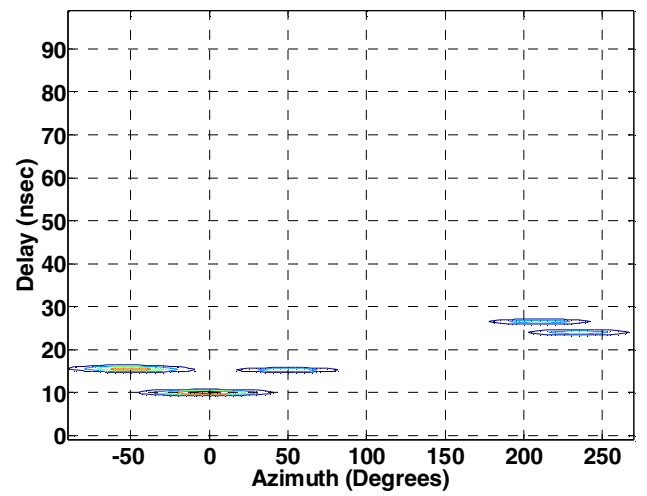

(b)

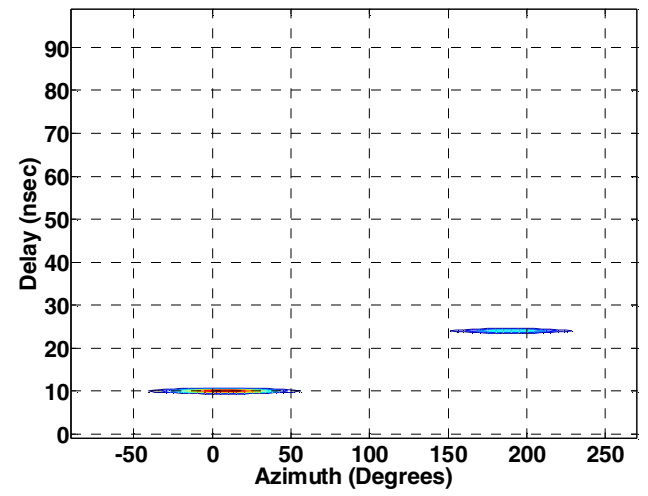

(c)

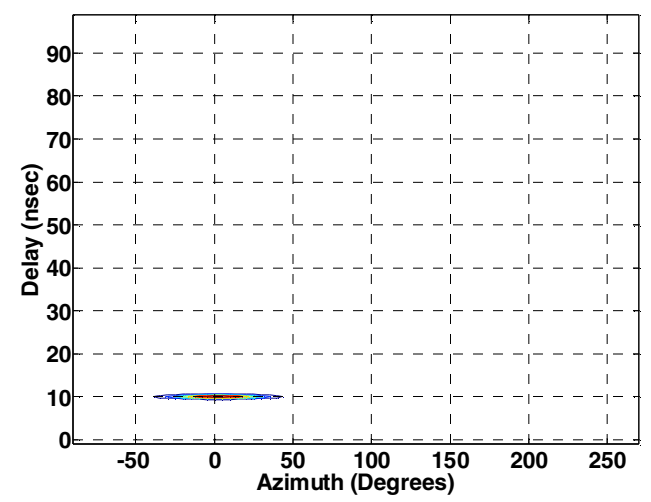

(d)

Figure 6: 2-D clusters (Residential LOS environment) (a) Omni (b) $\alpha=60^{\circ}$ (c) $\alpha=30^{\circ}$ (d) $\alpha=15^{\circ}$ (e.g. covered by electromagnetic absorbers in case of the transmitter) blocks the path of the reflected signal from either wall.

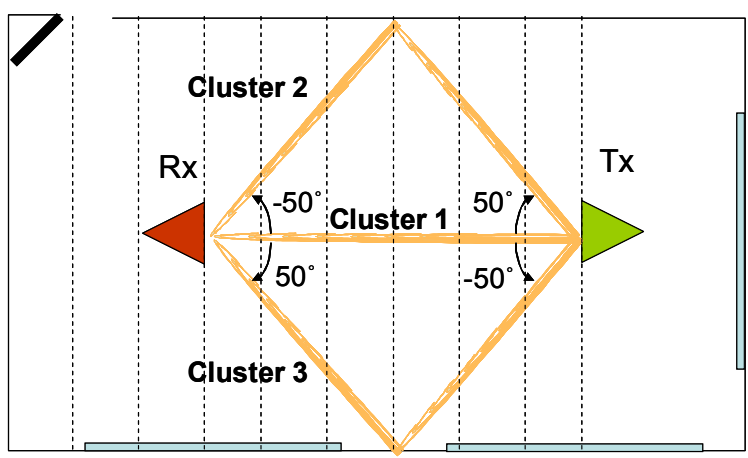

(a)

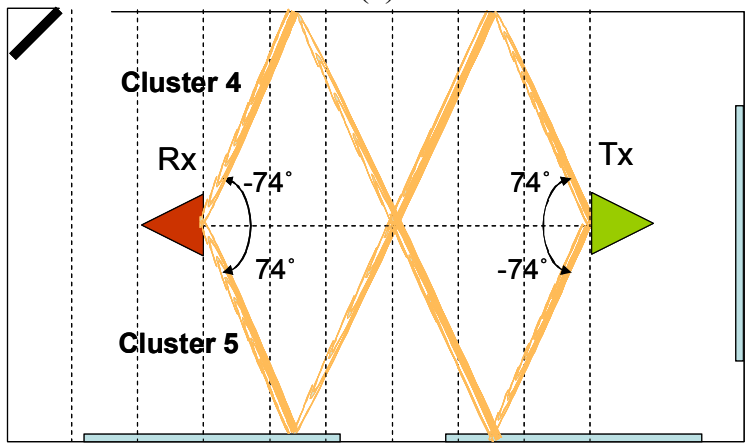

(b)

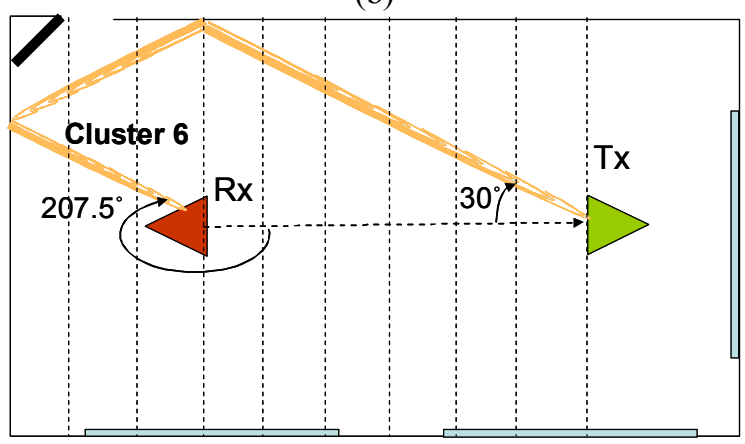

(c)

Figure 7: Radio paths resulting to clusters (a) 1,2,3 (b) 4,5 (c) 6

The plots indicating the cluster locations for the case when directional antennas with different beamwidths were used at the transmitter are shown in Figure 6. ' $\alpha$ ' represents the 3-dB beamwidth of the transmitting antenna. It is interesting to observe that as the beamwidth reduces, all clusters that occur due to paths outside the beamwidth of the antenna disappear. In other words, if the angle of departure of a path leading up to a cluster falls outside the beamwidth of the transmitter antenna, then the corresponding cluster will not be formed at the receiver. Also, looking at the case where $\alpha=60^{\circ}$ and compared to the omni-directional scenario, a new cluster (i.e. 7) is seen that is approximately located at $\left(231^{\circ}, 24.25 \mathrm{~ns}\right)$. Following the geometry of the layout, we conjecture that this cluster forms as a result of the reflection from the door as seen in Fig. 8. In the omni-directional case, the arrivals from this path are too weak and therefore not identifiable by the KDE. For $\alpha=15^{\circ}$, only the LOS cluster with minimal delay spread is observed. This is 
consistent with our expectation that as $\alpha$ gets smaller, channel behavior approaches an AWGN channel.

\section{B. NLOS Office Environment}

The data from the NLOS office environment was also processed for cluster identification. Figure 9 demonstrates the clusters that have been identified from the measured data. The scattering effect of the furniture and other office equipment in this environment causes some spreading in the shape of the clusters. For the omni-directional case, a total of 3 clusters are observed in channel data that are the results of the LOS and single-reflected paths.

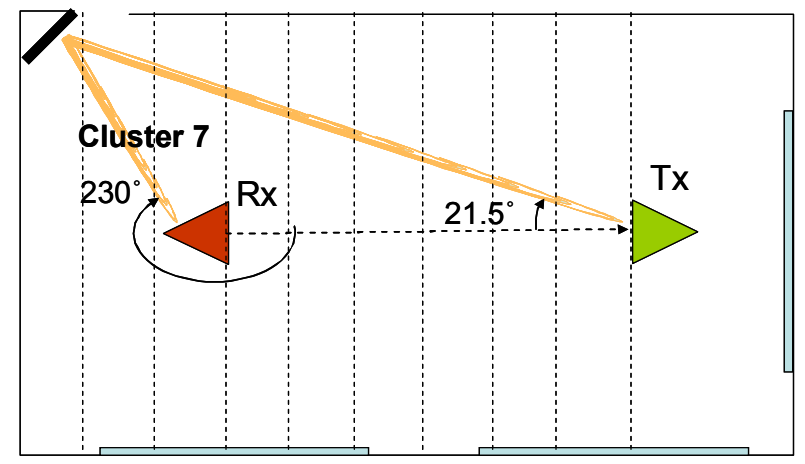

Figure 8: Radio path resulting to cluster 7

The coordinate of these clusters are listed in Table 2. The direct path between the transmitter and receiver, cluster 1 , and the arrivals due to the reflection from the metal wall, cluster2, have merged and formed a bigger cluster. The cluster 3 , occurs because of the single reflection from the metal wall behind the receiver.

Table 2: Cluster coordinates for the residential environments

\begin{tabular}{|c|c|c|}
\hline Cluster \# & $\begin{array}{c}\text { Cluster Arrival } \\
\text { Angle (Degrees) }\end{array}$ & $\begin{array}{c}\text { Cluster Arrival Time } \\
\text { (nsec) }\end{array}$ \\
\hline 1 & 0 & 37 \\
\hline 2 & -55 & 67.5 \\
\hline 3 & 178 & 117.5 \\
\hline
\end{tabular}

Looking at the time coordinate of some of these clusters, a mismatch is observed between the results and geometric calculation based on the RF paths. A physical explanation for this discrepancy can be given by noticing the velocity change of RF waves as it goes through different materials that exist on its path between the transmitter and receiver [13]. In general, the exact coordinate, shape and size of the clusters depends on the details of the propagation environments; however, as seen here, the locations of the main clusters (i.e. high density) can be reasonably approximated by using information about the layout and locations of the transmitter-receiver pair.

The clusters in figure 9 are due to the use of an omnidirectional antenna at the transmitter. If a directional antenna is used instead, arrivals become more localized in time-angle space and the cluster's sizes are reduced. The RF energy transmission in that case is more focused toward certain direction and therefore, the impact of the scattering on the ray arrivals is reduced. Figure 10 displays the clusters at the receiver when a directional antenna with $30^{\circ} 3-\mathrm{dB}$ bemawidth is used at the transmitter. The directional antenna is facing the receiver in this experiment.

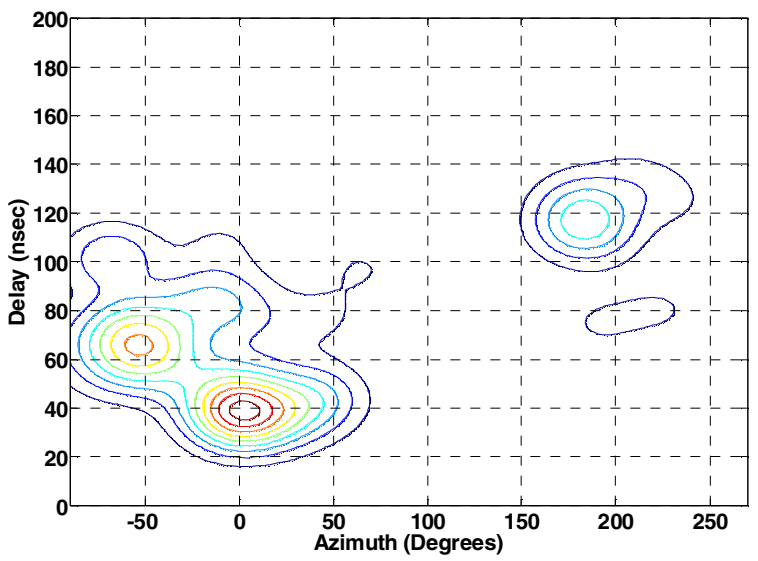

Figure 9: 2-D clusters (Office NLOS environment, Omni TX)

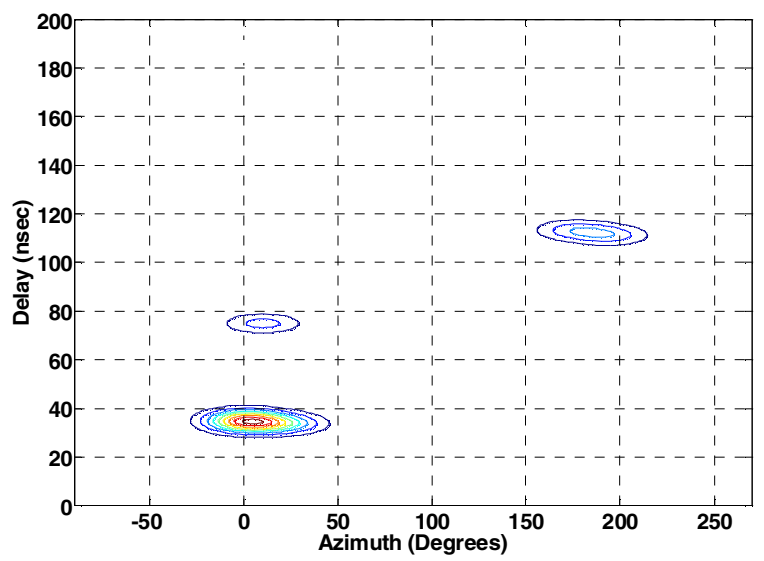

Figure 10: 2-D clusters (Office NLOS environment, directional TX)

\section{Cluster Energy}

We have also investigated energy distribution among the clusters in both residential and office environments. The normalized percentage of the received energy in each cluster is calculated as in [16], [17] and is referred to as the normalized relative energy.

Figures 11 and 12 illustrate these results for omni and directional antennas in residential LOS and office NLOS environments respectively. It is seen that high percentage of the energy is concentrated in the first cluster. This is especially expected in the LOS case where reflected rays travel longer path and therefore contribute less energy to their corresponding cluster at the receiver. This might not be the case for NLOS scenarios in general. Also as observed, using directional antennas with smaller beamwidths increases the energy of the $1^{\text {st }}$ cluster. This means that for such scenarios, a single cluster statistical model is probably sufficient to describe the channel behavior.

\section{CONCLUSION}

In this paper, we have discussed the clustering characteristics of a millimeter wave indoor channel, the influence of geometry on this phenomenon, and energy 
distribution among the clusters for directional antennas with various beamwidths. Knowledge of such spatial distribution of $\mathrm{RF}$ energy around the receiver in conjunction with array antennas and appropriate signal processing algorithms can lead to systems that provide superior performance by taking advantage of clusters location and their corresponding characteristics. Also, statistical models that contain both angular and temporal components of the millimeter wave indoor channel are needed to ensure more efficient design of millimeter wave systems. According to the energy distribution among the clusters, a single cluster statistical model is probably sufficient to describe the channel behavior in LOS scenarios. Further experimental results are required to verify and investigate this observation.

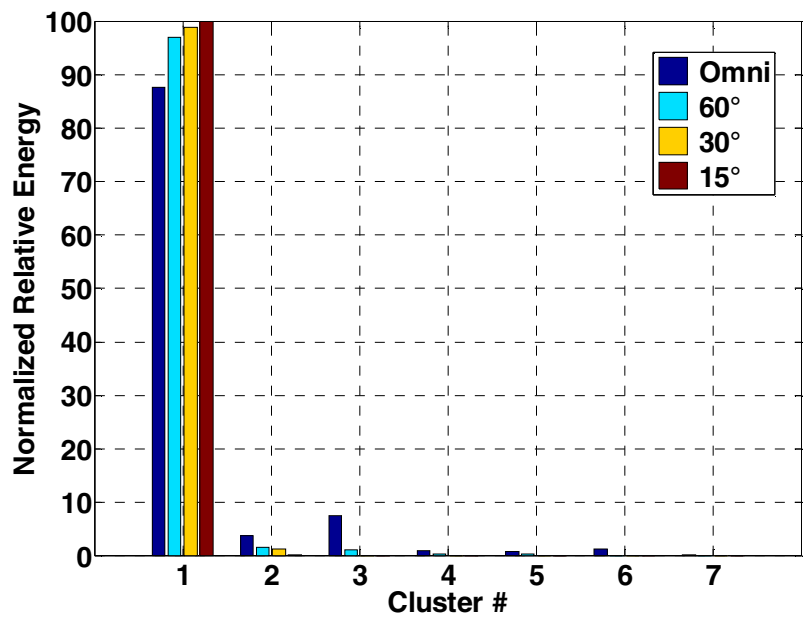

Figure 11: Normalized percentage of clusters energy for different transmitter 3-dB beamwidths (Residential LOS environment)

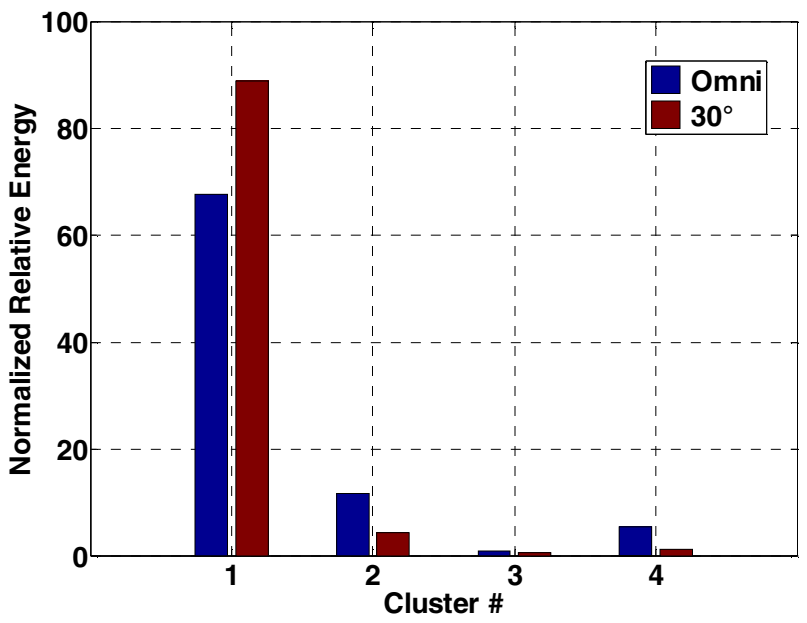

Figure 12: Normalized percentage of clusters energy for different 3-dB transmitter beamwidths (Office NLOS environment)

\section{ACKNOWLEDGMENT}

The authors would like to express their gratitude to Dr. Hirokazu Sawada, Dr. Yozo Shoji and Dr. Hiroyo Ogawa from the National Institute of Information and Communications Technology (NICT) in Japan for providing the measurement results. Also, authors would like to thank Dr. Quentin H. Spencer from Distributed Control Systems, Inc. for his valuable comments and insights regarding the CLEAN algorithm.

\section{REFERENCES}

[1] Q. H. Spencer, B. D. Jeffs, M. A. Jensen, A. L. Swindlehurst, "Modeling the statistical time and angle of arrival characteristics of an indoor multipath channel", IEEE Journal on Selected Areas in Communications, Vol. 18, No 3, Pages: 347 - 360, March 2000

[2] C. Chong, C. Tan, D. Laurenson, S. McLaughlin, M. A. Beach, A. R. Nix, "A New Statistical Wideband Spatio-Temporal Channel Model for 5GHz Band WLAN Systems", IEEE Journal on Selected Areas in Communications, Vol. 21, No. 2, February 2003

[3] H. Sawada, Y. Shoji, H. Ogawa, "Angle of Arrival Measurement in Home and Office Environments", National Institute of Information and Communications Technology (NICT), Japan, doc\# IEEE 802.15-060012-01-003c, IEEE 802.15-06-0013-01-003c

[4] S.M. Jefferies, J.C. Christou, "Restoration of Astronomical Images by Iterative Blind Deconvolution", Journal of Astrophy, No. 415, 1993

[5] L. B. Lucy, "An iterative technique for the rectification of observed distributions", The Astronomical Journal, Vol. 79, No. 6, 1974

[6] W. H. Richardson, "Bayesian-Based Iterative Method of Image Restoration", Journal of the Optical Society of America, Vol. 62, 1972

[7] J.A. Högbom, "Aperture synthesis with non-regular distribution of interferometer baselines" Astronomy and Astrophysics, Vol. 15, 1974

[8] A. Segalovitz, B. R. Frieden, "A CLEAN-type deconvolution algorithm", Astron. Astrophys., No. 70, Pages: 335-343, 1978

[9] D. G. Steer, P. E. Dewdney, M. R. Ito, "Enhancements to the deconvolution algorithm CLEAN", Astron. Astrophys., No. 137, 1984

[10] D. W. Scott, "Multivariate Density Estimation: Theory, Practice and Visualization" New York: Wiley, 1992.

[11] A. W. Bowman, A. Azzalini, "Applied Smoothing Techniques for Data Analysis, Oxford Univ. Press, 1997

[12] L. A. Thombs, S. J. Sheather, "Local Bandwidth Selection for Density Estimation" in the proceeding of the $22^{\text {nd }}$ Symposium on the Interface, page 111-116 Springer-Verlag, New York 1990

[13] M. A. Hussein, "Characterization of Ultra Wideband Communication Channels", Ph.D. Dissertation, Virginia Polytechnic Institute and State University, March 2003

[14] K. H. Li, M. A. Ingram, A. V. Nguyen, "Impact of clustering in statistical indoor propagation models on link capacity", IEEE Trans. on Communications, Vol. 50, Page(s): 521-523, April 2000

[15] K. Huang, Z. Wang, "Millimeter Wave Circular Polarized BeamSteering Antenna Array for Gigabit Wireless Communications", IEEE Trans. on Antennas and Propagation, Vol. 54, February 2006.

[16] Q. Spencer, M. Rice, B. Jeffs, and M. Jensen, "A statistical model for the angle-of-arrival in indoor multipath propagation," in Proc. IEEE Veh. Technol. Conf., 1997, pp. 1415-1419.

[17] R. J. Cramer, R. A. Scholtz, and M. Z. Win, "Evaluation of an UltraWide-Band Propagation Channel", IEEE Transaction on Antennas and Propagation, Vol. 50, No. 5, Pages: 561-570, May 2002

[18] B. Neekzad, K. Sayrafian-Pour, J. S. Baras, "Energy Efficient Millimeter Wave Radio Link Establishment for Low Probability of Intercept with Smart Antenna", $25^{\text {th }}$ Army Science Conference, Orlando, FL, November 2006

[19] B. Neekzad, K. Sayrafian-Pour, J. Perez, J. S. Baras, "Comparison of Ray Tracing Simulation and Millimeter Wave Channel Sounding Measurement", $18^{\text {th }}$ IEEE PIMRC, Athens, Greece, September 2007 